\title{
Properties of a Some (Ag-Cu-Sn) Alloys for Shielding Against Gamma Rays
}

\author{
M. A. M. Uosif \\ Physics Department, Faculty of Science, Al-Azher University, \\ Assuit Branch, 71524 Assuit, Egypt \\ Corresponding author: Fax: +20882181436-Mobil Tel.: +20/11/7058707 \\ Dr_Mohamed_Amin@Lycos.com
}

\begin{abstract}
The study of radiation absorption in material is an important subject to protect living creatures and environment from harmful effect of radiation. The degree to which gamma radiation is attenuated is dependent upon the energy of the incident gamma radiation, the thickness of the shielding, the atomic number and density of the elements in the shielding material. In this respect, this study presents the attenuating properties of some (Ag-Cu-Sn Alloys) by using the Nation Institute of Standards and Technology (NIST) XCOM code in the energy range (0.01 to $10 \mathrm{MeV})$.
\end{abstract}

Keywords: Mass attenuation coefficient; shielding material; Ag-Cu-Sn Alloys

\section{Introduction}

The word radiation was used until about 1900 to describe electromagnetic waves. Today, radiation refers to the whole electromagnetic spectrum as well as to the atomic and subatomic particles that have been discovered [1]. One of the many ways in which different types of radiation are grouped is in terms of ionizing and nonionizing radiation. The word ionizing refers to the ability to ionize an atom or a molecule of the medium it traverses.

The ionizing radiations are commonly classified into two principal types. Directly ionizing radiations include radiations of energetic particles carrying an electric charge, such as beta particles, alpha particles, protons, and other recoil nuclei. They cause ionization of direct action of electrons in atoms of the media through which they pass. Another type of radiation, indirectly ionizing such as neutrons and x-ray or gamma-ray photons are not charged and cause ionization through a more complicated mechanism involving the emission of energetic secondary charged particles which cause most of the ionization [2].

Directly ionizing radiation interacts very strongly with shielding media and is therefore easily stopped. By contrast, indirectly ionizing radiation, may be quite penetrating and the shielding required may be quite massive and expensive. For these reasons, nowadays much attention has been paid to the shielding of neutrons and photons, the two types of indirectly ionizing radiation most frequently encountered. Among all the types of radiation, this work involved gamma-ray.

Radiation shielding serves a number of functions [3] like:

1- Reducing the radiation exposure to persons in the vicinity of radiation sources.

2- Reducing the intensity of $\gamma$-rays incident on the reactor vessel.

3- Protecting some delicate electronic apparatus that otherwise would not function properly in a radiation shield, for example, in some types of military equipment. 
The effectiveness of the shielding material is determined by the interactions between the incident radiation and the atoms of the absorbing medium. The attenuation of gamma radiation occurs through the interaction of the gamma radiation with matter. Composite materials may offer additional benefits in chemical resistance, physical durability, and portability [4].

Shielding of gamma radiation primarily involves the interaction of gamma radiation with matter via three main processes [5] photoelectric effect, Compton scattering and pair production. Pair production occurs only for very high energy gamma rays $(>1022 \mathrm{keV})$.

The sum of (Photoelectric effect, Compton scattering and Pair production) probabilities is simply the probability per unit path length that the gamma-ray photon is removed from the beam is called the linear attenuation coefficient $(\mu)$ or macroscopic cross-section, which has the dimensions of inverse length $\left(\mathrm{cm}^{-1}\right)[6]$ :

$$
\mu=\sigma(\text { photoelectric })+\sigma(\text { Compton })+\sigma \text { (pair) }
$$

Historically, radiation shielding aprons and coverings has been manufactured from lead $(\mathrm{Pb})$. Early studies of non- $\mathrm{Pb}$ materials were prompted by the desire to reduce the weight of protective garments and the possibility of improved shielding performance [7]. Non- $\mathrm{Pb}$ protective materials can lower the total mass of a similar sized garment, providing a decrease in the mass of the garment while providing equivalent or better protection [8].

The purpose of this study is to determine the Gamma- Ray attenuation properties of some lead-free solder as (Ag-Cu-Sn) alloys, by using the Nation Institute of Standards and Technology (NIST) XCOM code in the energy range $(0.01$ to $10 \mathrm{MeV})$.

\section{Computing Procedure}

Photon Cross Sections Database (XCOM program) has been employed for calculating the photon cross sections for investigated alloys. The XCOM program provides total cross sections and attenuation coefficients as well as partial cross sections for incoherent scattering, coherent scattering, photoelectric absorption, and pair production in the field of the atomic nucleus and in the field of the atomic electrons [9]. XCOM program generates the cross sections and attenuation coefficients for elements, compounds and 84 mixtures, at energy between $1 \mathrm{keV}$ and $100 \mathrm{GeV}$.

The total cross sections and attenuation coefficients as well as partial cross sections for the following processes: incoherent scattering, coherent scattering, photoelectric absorption, and pair production in the field of the atomic nucleus and in the field of the atomic electrons [9]. The attenuation of gamma radiation (shielding) can be described by the following equation:

$$
\frac{I}{I_{o}}=e^{-\mu t}
$$

$\mathrm{I}=$ intensity after shielding, $\mathrm{I}_{\mathrm{o}}=$ incident intensity, $\mu\left(\mathrm{cm}^{-1}\right)=$ linear absorption coefficient and $\mathrm{t}$ $(\mathrm{cm})=$ thickness of absorber.

The fact that the linear attenuation coefficient varies with the density of the absorber limits its use, even if the absorber material is the same. Therefore, the mass attenuation coefficient $\left(\mu_{\rho}\right)$ is much more widely used and is defined as:

$$
\mu_{\mathrm{\rho}}=\frac{\boldsymbol{\mu}}{\boldsymbol{\rho}}\left(\mathrm{cm}^{2} \cdot \mathrm{gm}^{-1}\right)
$$


Where $(\rho)$ represents the density of the absorbing medium.

For compounds, the partial and total mass interaction coefficients, which are equal to the product of the corresponding cross sections times the number of target molecules per unit mass of the material.

The mass attenuation coefficient of a chemical compound or mixture can be calculated

$$
\text { from: } \quad \mu_{\rho=} \frac{\mu_{\rho}}{\rho}=\sum_{i} \omega_{i}\left(\frac{\mu_{i}}{\rho_{i}}\right)
$$

Where $\omega_{i}$ is the weight fraction of element $i$ in the compound or mixture.

The values of mass attenuation coefficients are dependent upon the absorption and scattering of the incident radiation caused by several different mechanisms, such as Rayleigh (coherent) scattering, Compton (incoherent scattering), Photoelectric Absorption, and Pair Production.

\section{Investigated Materials}

The physics of attenuation for all currently available shielding materials is now well documented. So in this work, we try to find new shielding materials can be lighter in weight than traditional shielding materials while providing similar gamma attenuation. For that reason we chose thirty three commercial $\mathrm{Ag}-\mathrm{Cu}-\mathrm{Sn}$ alloys for attenuation investigation. The properties of investigated alloys like composition, melting temperature, density and hardness are given in Table 1.

Table 1. The properties of the investigating alloys [10]

\begin{tabular}{|c|c|c|c|c|}
\hline Alloy Name & $\begin{array}{c}\text { Alloy composition } \\
\text { weight per cent }\end{array}$ & Melt. Temp. ${ }^{\circ} \mathrm{C}$ & $\operatorname{Density}\left(\mathrm{Mg} \mathrm{m}^{-3}\right)$ & $\begin{array}{c}\text { Hardness } \\
(\text { Hp) }\end{array}$ \\
\hline $\mathrm{Sn} 3.8 \mathrm{Ag} 0.7 \mathrm{Cu}$ & $\mathrm{Ag} 3.8 \mathrm{Cu} 0.7 \mathrm{Sn} 95.5$ & 217 & 7.5 & 15 \\
\hline $\mathrm{Sn} 0.5 \mathrm{Ag} 4 \mathrm{Cu}$ & Ag0.5Cu4Sn95.5 & 217 & 7.3 & ---------- \\
\hline $\mathrm{Sn} 3 \mathrm{Ag} 4 \mathrm{Cu}$ & $\mathrm{Ag} 3 \mathrm{Cu} 4 \mathrm{Sn} 93$ & 221 & 7.4 & ---------- \\
\hline $\mathrm{Sn} 3.6 \mathrm{Ag} 1 \mathrm{Cu}$ & $\mathrm{Ag} 3.6 \mathrm{Cu} 1 \mathrm{Sn} 95.4$ & 217 & $7.23[11]$ & --------- \\
\hline $\mathrm{Sn} 4.7 \mathrm{Ag} 1.7 \mathrm{Cu}$ & $\mathrm{Ag} 4.7 \mathrm{Cu} 1.7 \mathrm{Sn} 93.6$ & 217 & 7.4 & 10.25 \\
\hline $\mathrm{Sn} 3.5 \mathrm{Ag} 0.7 \mathrm{Cu}$ & $\mathrm{Ag} 3.5 \mathrm{Cu} 0.7 \mathrm{Sn} 95.8$ & 217.5 & $7.22[11]$ & --------- \\
\hline $\mathrm{Sn} 3.2 \mathrm{Ag} 0.8 \mathrm{Cu}$ & $\mathrm{Ag} 3.2 \mathrm{Cu} 0.8 \mathrm{Sn} 96$ & 217 & $7.22[11]$ & -------- \\
\hline $\mathrm{Sn} 3 \mathrm{Ag} 0.5 \mathrm{Cu}$ & $\mathrm{Ag} 3 \mathrm{Cu} 0.5 \mathrm{Sn} 96.5$ & 220 & $7.21[11]$ & ---------- \\
\hline $\mathrm{Sn} 3.2 \mathrm{Ag} 0.7 \mathrm{Cu}$ & $\mathrm{Ag} 3.2 \mathrm{Cu} 0.7 \mathrm{Sn} 96.1$ & 216 & 7.3 & --------- \\
\hline $\mathrm{Sn} 3.2 \mathrm{Ag} 0.5 \mathrm{Cu}$ & $\mathrm{Ag} 3.2 \mathrm{Cu} 0.5 \mathrm{Sn} 96.3$ & 217 & $7.21[11]$ & --------- \\
\hline $\mathrm{Sn} 3.5 \mathrm{Ag} 0.75 \mathrm{Cu}$ & $\mathrm{Ag} 3.5 \mathrm{Cu} 0.75 \mathrm{Sn} 95.75$ & 218 & 7.5 & -------- \\
\hline $\mathrm{Sn} 4 \mathrm{Ag} 0.5 \mathrm{Cu}$ & $\mathrm{Ag} 4 \mathrm{Cu} 0.5 \mathrm{Sn} 95.5$ & 217 & 7.39 & --------- \\
\hline $\mathrm{Sn} 4 \mathrm{Ag} 1 \mathrm{Cu}$ & $\mathrm{Ag} 4 \mathrm{Cu} 1 \mathrm{Sn} 95$ & 217 & $7.21[11]$ & --------- \\
\hline
\end{tabular}




\begin{tabular}{|c|c|c|c|c|}
\hline $\mathrm{Sn} 2 \mathrm{Ag} 0.75 \mathrm{Cu}$ & $\mathrm{Ag} 2 \mathrm{Cu} 0.75 \mathrm{Sn} 97.25$ & 217 & $7.19[11]$ & ---- \\
\hline $\mathrm{Sn} 3.6 \mathrm{Ag} 1.5 \mathrm{Cu}$ & Ag3.6Cu1.5Sn94.9 & 225 & $7.24[11]$ & --------- \\
\hline $\mathrm{Sn} 4.1 \mathrm{Ag} 0.9 \mathrm{Cu}$ & $\mathrm{Ag} 4.1 \mathrm{Cu} 0.9 \mathrm{Sn} 95$ & 216.5 & $7.24[11]$ & ---------- \\
\hline $\mathrm{Sn} 3.8 \mathrm{Ag} 2.3 \mathrm{Cu}$ & $\mathrm{Ag} 3.8 \mathrm{Cu} 2.3 \mathrm{Sn} 93.9$ & 217.4 & $7.25[11]$ & ---------- \\
\hline $\mathrm{Sn} 3.5 \mathrm{Ag} 1 \mathrm{Cu}$ & $\mathrm{Ag} 3.5 \mathrm{Cu} 1 \mathrm{Sn} 95.5$ & 216 & $7.23[11]$ & ---------- \\
\hline $\mathrm{Sn} 3.5 \mathrm{Ag} 1.3 \mathrm{Cu}$ & $\mathrm{Ag} 3.5 \mathrm{Cu} 1.3 \mathrm{Sn} 95.2$ & 216 & 7.4 & ---------- \\
\hline $\mathrm{Sn} 2.1 \mathrm{Ag} 0.9 \mathrm{Cu}$ & Ag2.1Cu0.9Sn97 & $216.9^{\circ} \mathrm{C}$ & $7.19[11]$ & 17.7 \\
\hline $\mathrm{Sn} 2.5 \mathrm{Ag} 0.9 \mathrm{Cu}$ & $\mathrm{Ag} 2.5 \mathrm{Cu} 0.9 \mathrm{Sn} 96.6$ & $216.8^{\circ} \mathrm{C}$ & $7.20[11]$ & 19.3 \\
\hline Sn $.95 \mathrm{Ag} 0.65 \mathrm{Cu}$ & Ag3.95Cu0.65Sn95.4 & $216.35^{\circ} \mathrm{C}$ & $7.23[11]$ & ---------- \\
\hline Sn $2.5 \mathrm{Ag} 0.7 \mathrm{Cu}$ & $\mathrm{Ag} 2.5 \mathrm{Cu} 0.7 \mathrm{Sn} 96.8$ & ---------- & $7.20[11]$ & --------- \\
\hline $\mathrm{Sn} 3.9 \mathrm{Ag} 0.6 \mathrm{Cu}$ & $\mathrm{Ag} 3.9 \mathrm{Cu} 0.6 \mathrm{Sn} 95.5$ & 217.5 & $7.23[11]$ & --------- \\
\hline $\mathrm{Sn} 2.0 \mathrm{Ag} 0.5 \mathrm{Cu}$ & $\mathrm{Ag} 2.0 \mathrm{Cu} 0.5 \mathrm{Sn} 97.5$ & ---------- & $7.18[11]$ & ---------- \\
\hline $\mathrm{Sn} 2.0 \mathrm{Ag} 1.5 \mathrm{Cu}$ & $\mathrm{Ag} 2.0 \mathrm{Cu} 1.5 \mathrm{Sn} 96.5$ & --------- & $7.20[11]$ & --------- \\
\hline $\mathrm{Sn} 3.5 \mathrm{Ag} 0.8 \mathrm{Cu}$ & Ag3.5Cu0.8Sn95.7 & ---------- & $7.22[11]$ & ---------- \\
\hline $\mathrm{Sn} 3.4 \mathrm{Ag} 0.9 \mathrm{Cu}$ & $\mathrm{Ag} 3.4 \mathrm{Cu} 0.9 \mathrm{Sn} 95.7$ & $217^{\circ} \mathrm{C}$ & $7.22[11]$ & ---------- \\
\hline $\mathrm{Sn} 3.0 \mathrm{Ag} 0.9 \mathrm{Cu}$ & $\mathrm{Ag} 3.0 \mathrm{Cu} 0.9 \mathrm{Sn} 96.1$ & $216.8^{\circ} \mathrm{C}$ & $7.21[11]$ & --------- \\
\hline $\mathrm{Sn} 2.0 \mathrm{Ag} 0.9 \mathrm{Cu}$ & Ag2.0Cu0.9Sn97.1 & $216.9^{\circ} \mathrm{C}$ & $7.19[11]$ & ---------- \\
\hline $\mathrm{Sn} 4.0 \mathrm{Ag} 1.5 \mathrm{Cu}$ & $\mathrm{Ag} 4 \mathrm{Cu} 1.5 \mathrm{Sn} 94.5$ & ---------- & $7.25[11]$ & -------- \\
\hline $\mathrm{Sn} 3.5 \mathrm{Ag} 0.5 \mathrm{Cu}$ & $\mathrm{Ag} 3.5 \mathrm{Cu} 1 \mathrm{Sn} 95.5$ & 205.15 & $7.23[11]$ & --------- \\
\hline $\mathrm{Sn} 3.5 \mathrm{Ag} 2.0 \mathrm{Cu}$ & $\mathrm{Ag} 3.5 \mathrm{Cu} 1 \mathrm{Sn} 95.5$ & 203.43 & $7.24[11]$ & --------- \\
\hline
\end{tabular}

The gamma radiation attenuation in shielding material is dependent upon the atomic number and density of that material. The density of investigated material (commercial Ag$\mathrm{Cu}-\mathrm{Sn}$ alloys) depends on the atomic number $(\mathrm{Z})$ and density $(\rho)$ on each of $(\mathrm{Ag})$ silver $(\mathrm{Z}=47$ and $\left.\rho=10.501 \mathrm{Mg} \cdot \mathrm{m}^{-3}\right),(\mathrm{Cu})$ copper $\left(\mathrm{Z}=29\right.$ and $\left.\rho=8.933 \mathrm{Mg} \cdot \mathrm{m}^{-3}\right)$ and $(\mathrm{Zn})$ zinc $(\mathrm{Z}=30$ and $\rho=$ $\left.7.134 \mathrm{Mg} \cdot \mathrm{m}^{-3}\right)$.

From the above data in Table 1 which are illustrated in Figure 1, it is clear that as silver and copper percentage increases, the density of the alloy increases. It was found that the highest alloy density is 7.5 Mg.m ${ }^{-3}$ for (Ag3.8 Cu0.7 Sn95.5) and (Ag3.5 Cu0.75 Sn95.75), while in case of $(\mathrm{Ag} 2.0 \mathrm{Cu} 0.5 \mathrm{Sn} 97.5)$ and $(\mathrm{Ag} 2.0 \mathrm{Cu} 0.9 \mathrm{Sn} 97.1)$ alloys which have low silver and copper percentage, its densities are 7.18 and $7.19 \mathrm{Mg} \cdot \mathrm{m}^{-1}$ respectively. Also from Figure 1, it is obvious that, the densities of all alloys are between 7.18 and $7.5 \mathrm{Mg} \cdot \mathrm{m}^{-1}$; this is due to that the majority of the composition of all alloys is tin with percentage between 93 to $97.25 \%$, which has a density of $7.134 \mathrm{Mg} \cdot \mathrm{m}^{-1}$. 


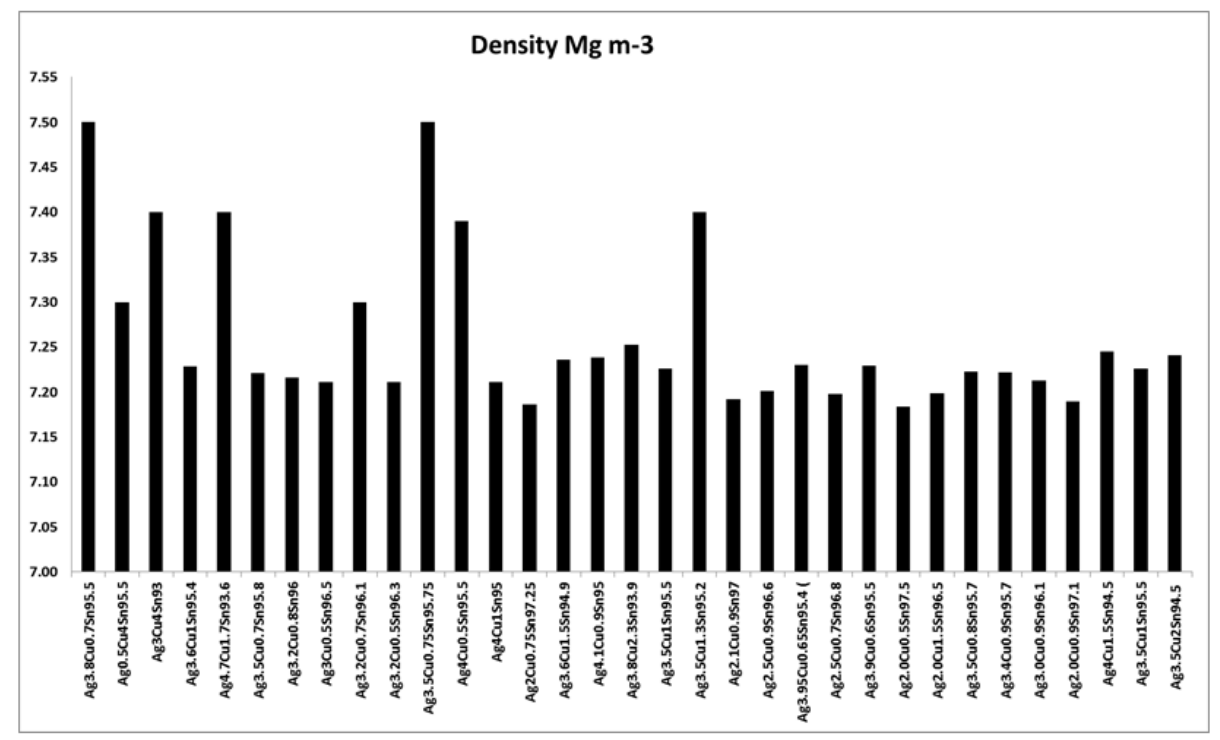

Figure 1. The density of all investigated alloys in $\mathrm{Mg} \cdot \mathrm{m}^{-1}$

\section{Results and Discussion}

The National Institute of Standards and Technology (NIST) XCOM program have been used to determine the attenuating properties of (Ag-Cu-Sn Alloys) samples in the energy range $(0.01$ to $10 \mathrm{MeV})$. The total mass absorption coefficients for the investigated alloys are obtained as sums of the corresponding quantities for the atomic constituents. The obtained results were listed in Table 2.

Table 2. Mass attenuation coefficient in $\left(\mathrm{cm}^{2} \cdot \mathrm{g}^{-1}\right)$ for all investigated alloys

\begin{tabular}{|c|c|c|c|c|c|c|}
\hline & \multicolumn{6}{|c|}{ Total mass attenuation coefficient $\left(\boldsymbol{\mu} \boldsymbol{)} \mathbf{~ c m}^{\mathbf{2}} \mathbf{. g}\right.$} \\
\hline Photon Energy(MeV) & Sn 3.8Ag0.7Cu & Sn 0.5Ag4Cu & Sn 3Ag4Cu & Sn 3.6Ag1Cu & Sn 4.7Ag1.7Cu & Sn 3.5Ag0.7Cu \\
\hline $1.00 \mathrm{E}-02$ & $1.38 \mathrm{E}+02$ & $1.41 \mathrm{E}+02$ & $\underline{\mathbf{1 . 4 1 E}+\mathbf{0 2}}$ & $1.39 \mathrm{E}+02$ & $1.39 \mathrm{E}+02$ & $1.38 \mathrm{E}+02$ \\
\hline $1.50 \mathrm{E}-02$ & $4.66 \mathrm{E}+01$ & $\mathbf{4 . 7 7 E + 0 1}$ & $4.75 \mathrm{E}+01$ & $4.67 \mathrm{E}+01$ & $4.68 \mathrm{E}+01$ & $4.66 \mathrm{E}+01$ \\
\hline $2.00 \mathrm{E}-02$ & $2.14 \mathrm{E}+01$ & $2.19 \mathrm{E}+01$ & $2.19 \mathrm{E}+01$ & $2.15 \mathrm{E}+01$ & $2.15 \mathrm{E}+01$ & $2.14 \mathrm{E}+01$ \\
\hline $2.55 \mathrm{E}-02$ & $1.11 \mathrm{E}+01$ & $1.14 \mathrm{E}+01$ & $1.13 \mathrm{E}+01$ & $1.11 \mathrm{E}+01$ & $1.12 \mathrm{E}+01$ & $1.11 \mathrm{E}+01$ \\
\hline $2.73 \mathrm{E}-02$ & $1.07 \mathrm{E}+01$ & $9.66 \mathrm{E}+00$ & $1.06 \mathrm{E}+01$ & $1.07 \mathrm{E}+01$ & $1.11 \mathrm{E}+01$ & $1.06 \mathrm{E}+01$ \\
\hline $2.92 \mathrm{E}-02$ & $8.99 \mathrm{E}+00$ & $8.08 \mathrm{E}+00$ & $8.87 \mathrm{E}+00$ & $8.94 \mathrm{E}+00$ & $9.31 \mathrm{E}+00$ & $8.89 \mathrm{E}+00$ \\
\hline $3.00 \mathrm{E}-02$ & $4.08 \mathrm{E}+01$ & $4.00 \mathrm{E}+01$ & $3.99 \mathrm{E}+01$ & $4.08 \mathrm{E}+01$ & $4.05 \mathrm{E}+01$ & $4.08 \mathrm{E}+01$ \\
\hline $4.00 \mathrm{E}-02$ & $1.92 \mathrm{E}+01$ & $1.88 \mathrm{E}+01$ & $1.88 \mathrm{E}+01$ & $1.92 \mathrm{E}+01$ & $1.91 \mathrm{E}+01$ & $1.93 \mathrm{E}+01$ \\
\hline $5.00 \mathrm{E}-02$ & $1.06 \mathrm{E}+01$ & $1.04 \mathrm{E}+01$ & $1.03 \mathrm{E}+01$ & $1.06 \mathrm{E}+01$ & $1.05 \mathrm{E}+01$ & $1.06 \mathrm{E}+01$ \\
\hline $6.00 \mathrm{E}-02$ & $6.50 \mathrm{E}+00$ & $6.36 \mathrm{E}+00$ & $6.34 \mathrm{E}+00$ & $6.49 \mathrm{E}+00$ & $6.44 \mathrm{E}+00$ & $6.50 \mathrm{E}+00$ \\
\hline $8.00 \mathrm{E}-02$ & $3.00 \mathrm{E}+00$ & $2.94 \mathrm{E}+00$ & $2.93 \mathrm{E}+00$ & $2.99 \mathrm{E}+00$ & $2.97 \mathrm{E}+00$ & $3.00 \mathrm{E}+00$ \\
\hline $1.00 \mathrm{E}-01$ & $1.66 \mathrm{E}+00$ & $1.63 \mathrm{E}+00$ & $1.62 \mathrm{E}+00$ & $1.66 \mathrm{E}+00$ & $1.65 \mathrm{E}+00$ & $1.66 \mathrm{E}+00$ \\
\hline $1.50 \mathrm{E}-01$ & $6.04 \mathrm{E}-01$ & $5.93 \mathrm{E}-01$ & $5.92 \mathrm{E}-01$ & $6.03 \mathrm{E}-01$ & $5.99 \mathrm{E}-01$ & $6.04 \mathrm{E}-01$ \\
\hline $2.00 \mathrm{E}-01$ & $3.24 \mathrm{E}-01$ & $3.19 \mathrm{E}-01$ & $3.18 \mathrm{E}-01$ & $3.23 \mathrm{E}-01$ & $3.22 \mathrm{E}-01$ & $3.24 \mathrm{E}-01$ \\
\hline $3.00 \mathrm{E}-01$ & $1.63 \mathrm{E}-01$ & $1.62 \mathrm{E}-01$ & $1.62 \mathrm{E}-01$ & $1.63 \mathrm{E}-01$ & $1.63 \mathrm{E}-01$ & $1.63 \mathrm{E}-01$ \\
\hline
\end{tabular}




\begin{tabular}{|c|c|c|c|c|c|c|}
\hline $4.00 \mathrm{E}-01$ & $1.15 \mathrm{E}-01$ & $1.15 \mathrm{E}-01$ & $1.15 \mathrm{E}-01$ & $1.15 \mathrm{E}-01$ & $1.15 \mathrm{E}-01$ & $1.15 \mathrm{E}-01$ \\
\hline $5.00 \mathrm{E}-01$ & $9.37 \mathrm{E}-02$ & $9.33 \mathrm{E}-02$ & $9.33 \mathrm{E}-02$ & $9.36 \mathrm{E}-02$ & $9.35 \mathrm{E}-02$ & $9.37 \mathrm{E}-02$ \\
\hline $6.00 \mathrm{E}-01$ & $8.11 \mathrm{E}-02$ & 8.09E-02 & $8.10 \mathrm{E}-02$ & $8.11 \mathrm{E}-02$ & $8.11 \mathrm{E}-02$ & $8.11 \mathrm{E}-02$ \\
\hline $8.00 \mathrm{E}-01$ & $6.67 \mathrm{E}-02$ & $6.66 \mathrm{E}-02$ & $6.66 \mathrm{E}-02$ & 6.67E-02 & 6.67E-02 & $6.67 \mathrm{E}-02$ \\
\hline $1.00 \mathrm{E}+00$ & $5.81 \mathrm{E}-02$ & $5.81 \mathrm{E}-02$ & $5.81 \mathrm{E}-02$ & $5.81 \mathrm{E}-02$ & $5.81 \mathrm{E}-02$ & $5.81 \mathrm{E}-02$ \\
\hline $1.02 \mathrm{E}+00$ & $5.74 \mathrm{E}-02$ & $5.74 \mathrm{E}-02$ & $5.74 \mathrm{E}-02$ & $5.74 \mathrm{E}-02$ & $5.74 \mathrm{E}-02$ & $5.74 \mathrm{E}-02$ \\
\hline $1.25 \mathrm{E}+00$ & $5.10 \mathrm{E}-02$ & $5.10 \mathrm{E}-02$ & $5.10 \mathrm{E}-02$ & $5.10 \mathrm{E}-02$ & $5.10 \mathrm{E}-02$ & $5.10 \mathrm{E}-02$ \\
\hline $1.50 \mathrm{E}+00$ & 4.64E-02 & 4.65E-02 & 4.65E-02 & 4.64E-02 & 4.65E-02 & 4.64E-02 \\
\hline $2.00 \mathrm{E}+00$ & 4.12E-02 & 4.12E-02 & 4.12E-02 & 4.12E-02 & 4.12E-02 & $4.12 \mathrm{E}-02$ \\
\hline $2.04 \mathrm{E}+00$ & 4.09E-02 & 4.09E-02 & 4.09E-02 & 4.09E-02 & 4.09E-02 & $4.09 \mathrm{E}-02$ \\
\hline $3.00 \mathrm{E}+00$ & $3.69 \mathrm{E}-02$ & $3.68 \mathrm{E}-02$ & 3.69E-02 & 3.69E-02 & $3.69 \mathrm{E}-02$ & $3.69 \mathrm{E}-02$ \\
\hline $4.00 \mathrm{E}+00$ & $3.56 \mathrm{E}-02$ & $3.55 \mathrm{E}-02$ & $3.55 \mathrm{E}-02$ & $3.56 \mathrm{E}-02$ & $3.56 \mathrm{E}-02$ & $3.56 \mathrm{E}-02$ \\
\hline $5.00 \mathrm{E}+00$ & $3.55 \mathrm{E}-02$ & $3.53 \mathrm{E}-02$ & $3.53 \mathrm{E}-02$ & $3.55 \mathrm{E}-02$ & $3.54 \mathrm{E}-02$ & $3.55 \mathrm{E}-02$ \\
\hline $6.00 \mathrm{E}+00$ & $3.58 \mathrm{E}-02$ & $3.56 \mathrm{E}-02$ & $3.57 \mathrm{E}-02$ & $3.58 \mathrm{E}-02$ & $3.58 \mathrm{E}-02$ & $3.58 \mathrm{E}-02$ \\
\hline $7.00 \mathrm{E}+00$ & $3.64 \mathrm{E}-02$ & $3.62 \mathrm{E}-02$ & $3.62 \mathrm{E}-02$ & $3.64 \mathrm{E}-02$ & $3.64 \mathrm{E}-02$ & $3.64 \mathrm{E}-02$ \\
\hline $8.00 \mathrm{E}+00$ & $3.72 \mathrm{E}-02$ & $3.70 \mathrm{E}-02$ & $3.70 \mathrm{E}-02$ & $3.72 \mathrm{E}-02$ & $3.71 \mathrm{E}-02$ & $3.72 \mathrm{E}-02$ \\
\hline $9.00 \mathrm{E}+00$ & $3.80 \mathrm{E}-02$ & $3.78 \mathrm{E}-02$ & $3.78 \mathrm{E}-02$ & $3.80 \mathrm{E}-02$ & $3.80 \mathrm{E}-02$ & $3.80 \mathrm{E}-02$ \\
\hline $1.00 \mathrm{E}+01$ & $3.89 \mathrm{E}-02$ & $3.86 \mathrm{E}-02$ & $3.86 \mathrm{E}-02$ & $3.89 \mathrm{E}-02$ & $3.88 \mathrm{E}-02$ & $3.89 \mathrm{E}-02$ \\
\hline Photon Energy(MeV) & Sn 3.2Ag0.8Cu & Sn $3 \mathrm{Ag} 0.5 \mathrm{Cu}$ & Sn $3.2 \mathrm{Ag} 0.7 \mathrm{Cu}$ & Sn $3.2 \mathrm{Ag} 0.5 \mathrm{Cu}$ & Sn 3.5Ag0.75Cu & Sn 4Ag0.5Cu \\
\hline $1.00 \mathrm{E}-02$ & $1.38 \mathrm{E}+02$ & $1.38 \mathrm{E}+02$ & $1.38 \mathrm{E}+02$ & $1.38 \mathrm{E}+02$ & $1.38 \mathrm{E}+02$ & $1.38 \mathrm{E}+02$ \\
\hline $1.50 \mathrm{E}-02$ & $4.67 \mathrm{E}+01$ & $4.66 \mathrm{E}+01$ & $4.66 \mathrm{E}+01$ & $4.66 \mathrm{E}+01$ & $4.66 \mathrm{E}+01$ & $4.65 \mathrm{E}+01$ \\
\hline $2.00 \mathrm{E}-02$ & $2.15 \mathrm{E}+01$ & $2.14 \mathrm{E}+01$ & $2.15 \mathrm{E}+01$ & $2.14 \mathrm{E}+01$ & $2.14 \mathrm{E}+01$ & $2.14 \mathrm{E}+01$ \\
\hline $2.55 \mathrm{E}-02$ & $1.11 \mathrm{E}+01$ & $1.11 \mathrm{E}+01$ & $1.11 \mathrm{E}+01$ & $1.11 \mathrm{E}+01$ & $1.11 \mathrm{E}+01$ & $1.11 \mathrm{E}+01$ \\
\hline 2.73E-02 & $1.05 \mathrm{E}+01$ & $1.04 \mathrm{E}+01$ & $1.05 \mathrm{E}+01$ & $1.05 \mathrm{E}+01$ & $1.06 \mathrm{E}+01$ & $1.08 \mathrm{E}+01$ \\
\hline 2.92E-02 & $8.80 \mathrm{E}+00$ & $8.73 \mathrm{E}+00$ & $8.80 \mathrm{E}+00$ & $8.79 \mathrm{E}+00$ & $8.89 \mathrm{E}+00$ & $9.04 \mathrm{E}+00$ \\
\hline $3.00 \mathrm{E}-02$ & $4.08 \mathrm{E}+01$ & $4.09 \mathrm{E}+01$ & $4.09 \mathrm{E}+01$ & $4.09 \mathrm{E}+01$ & $4.08 \mathrm{E}+01$ & $4.09 \mathrm{E}+01$ \\
\hline $4.00 \mathrm{E}-02$ & $1.92 \mathrm{E}+01$ & $1.93 \mathrm{E}+01$ & $1.93 \mathrm{E}+01$ & $1.93 \mathrm{E}+01$ & $1.92 \mathrm{E}+01$ & $1.93 \mathrm{E}+01$ \\
\hline $5.00 \mathrm{E}-02$ & $1.06 \mathrm{E}+01$ & $1.06 \mathrm{E}+01$ & $1.06 \mathrm{E}+01$ & $1.06 \mathrm{E}+01$ & $1.06 \mathrm{E}+01$ & $1.06 \mathrm{E}+01$ \\
\hline $6.00 \mathrm{E}-02$ & $6.50 \mathrm{E}+00$ & $6.52 \mathrm{E}+00$ & $6.51 \mathrm{E}+00$ & $6.52 \mathrm{E}+00$ & $6.50 \mathrm{E}+00$ & $6.51 \mathrm{E}+00$ \\
\hline $8.00 \mathrm{E}-02$ & $3.00 \mathrm{E}+00$ & $3.01 \mathrm{E}+00$ & $3.00 \mathrm{E}+00$ & $3.01 \mathrm{E}+00$ & $3.00 \mathrm{E}+00$ & $3.00 \mathrm{E}+00$ \\
\hline $1.00 \mathrm{E}-01$ & $1.66 \mathrm{E}+00$ & $1.66 \mathrm{E}+00$ & $1.66 \mathrm{E}+00$ & $1.66 \mathrm{E}+00$ & $1.66 \mathrm{E}+00$ & $1.66 \mathrm{E}+00$ \\
\hline $1.50 \mathrm{E}-01$ & $6.04 \mathrm{E}-01$ & $6.05 \mathrm{E}-01$ & $6.04 \mathrm{E}-01$ & $6.05 \mathrm{E}-01$ & $6.04 \mathrm{E}-01$ & $6.05 \mathrm{E}-01$ \\
\hline $2.00 \mathrm{E}-01$ & 3.24E-01 & 3.24E-01 & 3.24E-01 & $3.24 \mathrm{E}-01$ & 3.24E-01 & 3.24E-01 \\
\hline $3.00 \mathrm{E}-01$ & $1.63 \mathrm{E}-01$ & $1.63 \mathrm{E}-01$ & $1.63 \mathrm{E}-01$ & $1.63 \mathrm{E}-01$ & $1.63 \mathrm{E}-01$ & $1.63 \mathrm{E}-01$ \\
\hline $4.00 \mathrm{E}-01$ & $1.15 \mathrm{E}-01$ & $1.15 \mathrm{E}-01$ & $1.15 \mathrm{E}-01$ & $1.15 \mathrm{E}-01$ & $1.15 \mathrm{E}-01$ & $1.15 \mathrm{E}-01$ \\
\hline $5.00 \mathrm{E}-01$ & $9.36 \mathrm{E}-02$ & $9.37 \mathrm{E}-02$ & $9.37 \mathrm{E}-02$ & $9.37 \mathrm{E}-02$ & $9.36 \mathrm{E}-02$ & 9.37E-02 \\
\hline $6.00 \mathrm{E}-01$ & $8.11 \mathrm{E}-02$ & $8.11 \mathrm{E}-02$ & $8.11 \mathrm{E}-02$ & $8.11 \mathrm{E}-02$ & $8.11 \mathrm{E}-02$ & $8.11 \mathrm{E}-02$ \\
\hline $8.00 \mathrm{E}-01$ & $6.67 \mathrm{E}-02$ & $6.67 \mathrm{E}-02$ & $6.67 \mathrm{E}-02$ & $6.67 \mathrm{E}-02$ & $6.67 \mathrm{E}-02$ & 6.67E-02 \\
\hline $1.00 \mathrm{E}+00$ & $5.81 \mathrm{E}-02$ & $5.80 \mathrm{E}-02$ & $5.81 \mathrm{E}-02$ & $5.81 \mathrm{E}-02$ & $5.81 \mathrm{E}-02$ & $5.81 \mathrm{E}-02$ \\
\hline $1.02 \mathrm{E}+00$ & $5.74 \mathrm{E}-02$ & $5.73 \mathrm{E}-02$ & $5.74 \mathrm{E}-02$ & $5.73 \mathrm{E}-02$ & $5.74 \mathrm{E}-02$ & $5.74 \mathrm{E}-02$ \\
\hline
\end{tabular}




\begin{tabular}{|c|c|c|c|c|c|c|}
\hline $1.25 \mathrm{E}+00$ & 5.10E-02 & $5.10 \mathrm{E}-02$ & $5.10 \mathrm{E}-02$ & $5.10 \mathrm{E}-02$ & $5.10 \mathrm{E}-02$ & $5.10 \mathrm{E}-02$ \\
\hline $1.50 \mathrm{E}+00$ & 4.64E-02 & 4.64E-02 & 4.64E-02 & 4.64E-02 & 4.64E-02 & $4.64 \mathrm{E}-02$ \\
\hline $2.00 \mathrm{E}+00$ & 4.12E-02 & 4.12E-02 & 4.12E-02 & 4.12E-02 & 4.12E-02 & $4.12 \mathrm{E}-02$ \\
\hline $2.04 \mathrm{E}+00$ & 4.09E-02 & 4.09E-02 & 4.09E-02 & 4.09E-02 & 4.09E-02 & 4.09E-02 \\
\hline $3.00 \mathrm{E}+00$ & $3.69 \mathrm{E}-02$ & $3.69 \mathrm{E}-02$ & $3.69 \mathrm{E}-02$ & $3.69 \mathrm{E}-02$ & $3.69 \mathrm{E}-02$ & $3.69 \mathrm{E}-02$ \\
\hline $4.00 \mathrm{E}+00$ & $3.56 \mathrm{E}-02$ & $3.56 \mathrm{E}-02$ & $3.56 \mathrm{E}-02$ & $3.56 \mathrm{E}-02$ & $3.56 \mathrm{E}-02$ & $3.56 \mathrm{E}-02$ \\
\hline $5.00 \mathrm{E}+00$ & $3.55 \mathrm{E}-02$ & $3.55 \mathrm{E}-02$ & $3.55 \mathrm{E}-02$ & $3.55 \mathrm{E}-02$ & $3.55 \mathrm{E}-02$ & $3.55 \mathrm{E}-02$ \\
\hline $6.00 \mathrm{E}+00$ & $3.58 \mathrm{E}-02$ & $3.58 \mathrm{E}-02$ & $3.58 \mathrm{E}-02$ & $3.58 \mathrm{E}-02$ & $3.58 \mathrm{E}-02$ & $3.58 \mathrm{E}-02$ \\
\hline $7.00 \mathrm{E}+00$ & 3.64E-02 & 3.64E-02 & 3.64E-02 & 3.64E-02 & 3.64E-02 & $3.64 \mathrm{E}-02$ \\
\hline $8.00 \mathrm{E}+00$ & $3.72 \mathrm{E}-02$ & $3.72 \mathrm{E}-02$ & $3.72 \mathrm{E}-02$ & $3.72 \mathrm{E}-02$ & $3.72 \mathrm{E}-02$ & $3.72 \mathrm{E}-02$ \\
\hline $9.00 \mathrm{E}+00$ & $3.80 \mathrm{E}-02$ & $3.80 \mathrm{E}-02$ & $3.80 \mathrm{E}-02$ & $3.80 \mathrm{E}-02$ & $3.80 \mathrm{E}-02$ & $3.80 \mathrm{E}-02$ \\
\hline $1.00 \mathrm{E}+01$ & $3.89 \mathrm{E}-02$ & $3.89 \mathrm{E}-02$ & $3.89 \mathrm{E}-02$ & $3.89 \mathrm{E}-02$ & $3.89 \mathrm{E}-02$ & $3.89 \mathrm{E}-02$ \\
\hline Photon Energy(MeV) & Sn 4Ag1Cu & Sn $2 \mathrm{Ag} 0.75 \mathrm{Cu}$ & Sn 3.6Ag1.5Cu & Sn $4.1 \mathrm{Ag} 0.9 \mathrm{Cu}$ & Sn 3.8Ag2.3Cu & Sn 3.5Ag1Cu \\
\hline $1.00 \mathrm{E}-02$ & $1.38 \mathrm{E}+02$ & $1.39 \mathrm{E}+02$ & $1.39 \mathrm{E}+02$ & $1.38 \mathrm{E}+02$ & $1.40 \mathrm{E}+02$ & $1.39 \mathrm{E}+02$ \\
\hline $1.50 \mathrm{E}-02$ & 4.67E+01 & $4.67 \mathrm{E}+01$ & $4.68 \mathrm{E}+01$ & $4.66 \mathrm{E}+01$ & $4.70 \mathrm{E}+01$ & $4.67 \mathrm{E}+01$ \\
\hline $2.00 \mathrm{E}-02$ & $2.15 \mathrm{E}+01$ & $2.15 \mathrm{E}+01$ & $2.15 \mathrm{E}+01$ & $2.14 \mathrm{E}+01$ & $2.16 \mathrm{E}+01$ & $2.15 \mathrm{E}+01$ \\
\hline $2.55 \mathrm{E}-02$ & $1.11 \mathrm{E}+01$ & $1.12 \mathrm{E}+01$ & $1.12 \mathrm{E}+01$ & $1.11 \mathrm{E}+01$ & $1.12 \mathrm{E}+01$ & $1.11 \mathrm{E}+01$ \\
\hline $2.73 \mathrm{E}-02$ & $1.08 \mathrm{E}+01$ & $1.01 \mathrm{E}+01$ & $1.07 \mathrm{E}+01$ & $1.09 \mathrm{E}+01$ & $1.08 \mathrm{E}+01$ & $1.06 \mathrm{E}+01$ \\
\hline 2.92E-02 & $9.06 \mathrm{E}+00$ & $8.42 \mathrm{E}+00$ & $8.96 \mathrm{E}+00$ & $9.09 \mathrm{E}+00$ & $9.05 \mathrm{E}+00$ & $8.90 \mathrm{E}+00$ \\
\hline $3.00 \mathrm{E}-02$ & 4.07E+01 & $4.09 \mathrm{E}+01$ & $4.06 \mathrm{E}+01$ & $4.08 \mathrm{E}+01$ & $4.03 \mathrm{E}+01$ & $4.08 \mathrm{E}+01$ \\
\hline $4.00 \mathrm{E}-02$ & $1.92 \mathrm{E}+01$ & $1.93 \mathrm{E}+01$ & $1.91 \mathrm{E}+01$ & $1.92 \mathrm{E}+01$ & $1.90 \mathrm{E}+01$ & $1.92 \mathrm{E}+01$ \\
\hline $5.00 \mathrm{E}-02$ & $1.06 \mathrm{E}+01$ & $1.06 \mathrm{E}+01$ & $1.05 \mathrm{E}+01$ & $1.06 \mathrm{E}+01$ & $1.05 \mathrm{E}+01$ & $1.06 \mathrm{E}+01$ \\
\hline $6.00 \mathrm{E}-02$ & $6.48 \mathrm{E}+00$ & $6.51 \mathrm{E}+00$ & $6.46 \mathrm{E}+00$ & $6.49 \mathrm{E}+00$ & $6.42 \mathrm{E}+00$ & $6.49 \mathrm{E}+00$ \\
\hline $8.00 \mathrm{E}-02$ & $2.99 \mathrm{E}+00$ & $3.00 \mathrm{E}+00$ & $2.98 \mathrm{E}+00$ & $2.99 \mathrm{E}+00$ & $2.96 \mathrm{E}+00$ & $2.99 \mathrm{E}+00$ \\
\hline $1.00 \mathrm{E}-01$ & $1.66 \mathrm{E}+00$ & $1.66 \mathrm{E}+00$ & $1.65 \mathrm{E}+00$ & $1.66 \mathrm{E}+00$ & $1.64 \mathrm{E}+00$ & $1.66 \mathrm{E}+00$ \\
\hline $1.50 \mathrm{E}-01$ & $6.03 \mathrm{E}-01$ & $6.05 \mathrm{E}-01$ & $6.01 \mathrm{E}-01$ & $6.03 \mathrm{E}-01$ & $5.98 \mathrm{E}-01$ & $6.03 \mathrm{E}-01$ \\
\hline $2.00 \mathrm{E}-01$ & 3.23E-01 & 3.24E-01 & 3.22E-01 & 3.23E-01 & $3.21 \mathrm{E}-01$ & $3.23 \mathrm{E}-01$ \\
\hline $3.00 \mathrm{E}-01$ & $1.63 \mathrm{E}-01$ & $1.63 \mathrm{E}-01$ & $1.63 \mathrm{E}-01$ & $1.63 \mathrm{E}-01$ & $1.62 \mathrm{E}-01$ & $1.63 \mathrm{E}-01$ \\
\hline $4.00 \mathrm{E}-01$ & 1.15E-01 & $1.15 \mathrm{E}-01$ & $1.15 \mathrm{E}-01$ & $1.15 \mathrm{E}-01$ & $1.15 \mathrm{E}-01$ & $1.15 \mathrm{E}-01$ \\
\hline $5.00 \mathrm{E}-01$ & $9.36 \mathrm{E}-02$ & $9.37 \mathrm{E}-02$ & $9.36 \mathrm{E}-02$ & $9.36 \mathrm{E}-02$ & $9.35 \mathrm{E}-02$ & $9.36 \mathrm{E}-02$ \\
\hline $6.00 \mathrm{E}-01$ & $8.11 \mathrm{E}-02$ & $8.11 \mathrm{E}-02$ & $8.11 \mathrm{E}-02$ & $8.11 \mathrm{E}-02$ & $8.10 \mathrm{E}-02$ & $8.11 \mathrm{E}-02$ \\
\hline $8.00 \mathrm{E}-01$ & 6.67E-02 & $6.66 \mathrm{E}-02$ & $6.67 \mathrm{E}-02$ & 6.67E-02 & $6.67 \mathrm{E}-02$ & $6.67 \mathrm{E}-02$ \\
\hline $1.00 \mathrm{E}+00$ & $5.81 \mathrm{E}-02$ & $5.80 \mathrm{E}-02$ & $5.81 \mathrm{E}-02$ & $5.81 \mathrm{E}-02$ & $5.81 \mathrm{E}-02$ & $5.81 \mathrm{E}-02$ \\
\hline $1.02 \mathrm{E}+00$ & $5.74 \mathrm{E}-02$ & $5.73 \mathrm{E}-02$ & $5.74 \mathrm{E}-02$ & $5.74 \mathrm{E}-02$ & $5.74 \mathrm{E}-02$ & $5.74 \mathrm{E}-02$ \\
\hline $1.25 \mathrm{E}+00$ & $5.10 \mathrm{E}-02$ & $5.10 \mathrm{E}-02$ & $5.10 \mathrm{E}-02$ & $5.10 \mathrm{E}-02$ & $5.10 \mathrm{E}-02$ & $5.10 \mathrm{E}-02$ \\
\hline $1.50 \mathrm{E}+00$ & $4.65 \mathrm{E}-02$ & 4.64E-02 & 4.65E-02 & 4.65E-02 & $4.65 \mathrm{E}-02$ & 4.64E-02 \\
\hline $2.00 \mathrm{E}+00$ & 4.12E-02 & 4.12E-02 & 4.12E-02 & 4.12E-02 & 4.12E-02 & $4.12 \mathrm{E}-02$ \\
\hline $2.04 \mathrm{E}+00$ & $4.09 \mathrm{E}-02$ & $4.09 \mathrm{E}-02$ & $4.09 \mathrm{E}-02$ & $4.09 \mathrm{E}-02$ & $4.09 \mathrm{E}-02$ & $4.09 \mathrm{E}-02$ \\
\hline $3.00 \mathrm{E}+00$ & $3.69 \mathrm{E}-02$ & $3.69 \mathrm{E}-02$ & 3.69E-02 & 3.69E-02 & $3.69 \mathrm{E}-02$ & $3.69 \mathrm{E}-02$ \\
\hline $4.00 \mathrm{E}+00$ & $3.56 \mathrm{E}-02$ & $3.56 \mathrm{E}-02$ & $3.56 \mathrm{E}-02$ & $3.56 \mathrm{E}-02$ & $3.56 \mathrm{E}-02$ & $3.56 \mathrm{E}-02$ \\
\hline
\end{tabular}




\begin{tabular}{|c|c|c|c|c|c|c|}
\hline $5.00 \mathrm{E}+00$ & $3.55 \mathrm{E}-02$ & $3.55 \mathrm{E}-02$ & $3.54 \mathrm{E}-02$ & $3.55 \mathrm{E}-02$ & $3.54 \mathrm{E}-02$ & $3.55 \mathrm{E}-02$ \\
\hline $6.00 \mathrm{E}+00$ & $3.58 \mathrm{E}-02$ & $3.58 \mathrm{E}-02$ & $3.58 \mathrm{E}-02$ & $3.58 \mathrm{E}-02$ & $3.57 \mathrm{E}-02$ & $3.58 \mathrm{E}-02$ \\
\hline $7.00 \mathrm{E}+00$ & $3.64 \mathrm{E}-02$ & $3.64 \mathrm{E}-02$ & $3.64 \mathrm{E}-02$ & $3.64 \mathrm{E}-02$ & $3.63 \mathrm{E}-02$ & $3.64 \mathrm{E}-02$ \\
\hline $8.00 \mathrm{E}+00$ & $3.72 \mathrm{E}-02$ & 3.72E-02 & $3.71 \mathrm{E}-02$ & $3.72 \mathrm{E}-02$ & $3.71 \mathrm{E}-02$ & $3.72 \mathrm{E}-02$ \\
\hline $9.00 \mathrm{E}+00$ & $3.80 \mathrm{E}-02$ & $3.80 \mathrm{E}-02$ & $3.80 \mathrm{E}-02$ & $3.80 \mathrm{E}-02$ & $3.79 \mathrm{E}-02$ & $3.80 \mathrm{E}-02$ \\
\hline $1.00 \mathrm{E}+01$ & $3.89 \mathrm{E}-02$ & $3.89 \mathrm{E}-02$ & $3.88 \mathrm{E}-02$ & $3.89 \mathrm{E}-02$ & $3.88 \mathrm{E}-02$ & $3.89 \mathrm{E}-02$ \\
\hline Photon Energy(MeV) & Sn 3.5Ag1.3Cu & Sn 2.1Ag0.9Cu & Sn 2.5Ag0.9Cu & Sn $3.95 \mathrm{Ag} 0.65 \mathrm{Cu}$ & Sn 2.5Ag0.7Cu & Sn 3.9Ag0.6Cu \\
\hline $1.00 \mathrm{E}-02$ & $1.39 \mathrm{E}+02$ & $1.39 \mathrm{E}+02$ & $1.39 \mathrm{E}+02$ & $1.38 \mathrm{E}+02$ & $1.39 \mathrm{E}+02$ & $1.38 \mathrm{E}+02$ \\
\hline $1.50 \mathrm{E}-02$ & $4.68 \mathrm{E}+01$ & $4.68 \mathrm{E}+01$ & $4.67 \mathrm{E}+01$ & $4.66 \mathrm{E}+01$ & $4.67 \mathrm{E}+01$ & $4.66 \mathrm{E}+01$ \\
\hline $2.00 \mathrm{E}-02$ & $2.15 \mathrm{E}+01$ & $2.15 \mathrm{E}+01$ & $2.15 \mathrm{E}+01$ & $2.14 \mathrm{E}+01$ & $2.15 \mathrm{E}+01$ & $2.14 \mathrm{E}+01$ \\
\hline $2.55 \mathrm{E}-02$ & $1.12 \mathrm{E}+01$ & $1.12 \mathrm{E}+01$ & $1.12 \mathrm{E}+01$ & $1.11 \mathrm{E}+01$ & $1.11 \mathrm{E}+01$ & $1.11 \mathrm{E}+01$ \\
\hline $2.73 \mathrm{E}-02$ & $1.07 \mathrm{E}+01$ & $1.01 \mathrm{E}+01$ & $1.03 \mathrm{E}+01$ & $1.08 \mathrm{E}+01$ & $1.03 \mathrm{E}+01$ & $1.08 \mathrm{E}+01$ \\
\hline $2.92 \mathrm{E}-02$ & $8.92 \mathrm{E}+00$ & $8.46 \mathrm{E}+00$ & $8.58 \mathrm{E}+00$ & $9.03 \mathrm{E}+00$ & $8.58 \mathrm{E}+00$ & $9.01 \mathrm{E}+00$ \\
\hline $3.00 \mathrm{E}-02$ & $4.07 \mathrm{E}+01$ & $4.09 \mathrm{E}+01$ & $4.08 \mathrm{E}+01$ & $4.08 \mathrm{E}+01$ & $4.09 \mathrm{E}+01$ & $4.09 \mathrm{E}+01$ \\
\hline $4.00 \mathrm{E}-02$ & $1.92 \mathrm{E}+01$ & $1.93 \mathrm{E}+01$ & $1.92 \mathrm{E}+01$ & $1.92 \mathrm{E}+01$ & $1.93 \mathrm{E}+01$ & $1.93 \mathrm{E}+01$ \\
\hline $5.00 \mathrm{E}-02$ & $1.06 \mathrm{E}+01$ & $1.06 \mathrm{E}+01$ & $1.06 \mathrm{E}+01$ & $1.06 \mathrm{E}+01$ & $1.06 \mathrm{E}+01$ & $1.06 \mathrm{E}+01$ \\
\hline $6.00 \mathrm{E}-02$ & $6.47 \mathrm{E}+00$ & $6.50 \mathrm{E}+00$ & $6.50 \mathrm{E}+00$ & $6.50 \mathrm{E}+00$ & $6.51 \mathrm{E}+00$ & $6.50 \mathrm{E}+00$ \\
\hline $8.00 \mathrm{E}-02$ & $2.99 \mathrm{E}+00$ & $3.00 \mathrm{E}+00$ & $3.00 \mathrm{E}+00$ & $3.00 \mathrm{E}+00$ & $3.00 \mathrm{E}+00$ & $3.00 \mathrm{E}+00$ \\
\hline $1.00 \mathrm{E}-01$ & $1.65 \mathrm{E}+00$ & $1.66 \mathrm{E}+00$ & $1.66 \mathrm{E}+00$ & $1.66 \mathrm{E}+00$ & $1.66 \mathrm{E}+00$ & $1.66 \mathrm{E}+00$ \\
\hline $1.50 \mathrm{E}-01$ & $6.02 \mathrm{E}-01$ & $6.04 \mathrm{E}-01$ & 6.04E-01 & 6.04E-01 & $6.05 \mathrm{E}-01$ & 6.04E-01 \\
\hline $2.00 \mathrm{E}-01$ & $3.23 \mathrm{E}-01$ & $3.24 \mathrm{E}-01$ & $3.24 \mathrm{E}-01$ & $3.24 \mathrm{E}-01$ & $3.24 \mathrm{E}-01$ & $3.24 \mathrm{E}-01$ \\
\hline $3.00 \mathrm{E}-01$ & $1.63 \mathrm{E}-01$ & $1.63 \mathrm{E}-01$ & $1.63 \mathrm{E}-01$ & $1.63 \mathrm{E}-01$ & $1.63 \mathrm{E}-01$ & $1.63 \mathrm{E}-01$ \\
\hline $4.00 \mathrm{E}-01$ & $1.15 \mathrm{E}-01$ & $1.15 \mathrm{E}-01$ & $1.15 \mathrm{E}-01$ & $1.15 \mathrm{E}-01$ & $1.15 \mathrm{E}-01$ & $1.15 \mathrm{E}-01$ \\
\hline $5.00 \mathrm{E}-01$ & $9.36 \mathrm{E}-02$ & $9.36 \mathrm{E}-02$ & $9.36 \mathrm{E}-02$ & $9.37 \mathrm{E}-02$ & $9.37 \mathrm{E}-02$ & $9.37 \mathrm{E}-02$ \\
\hline $6.00 \mathrm{E}-01$ & $8.11 \mathrm{E}-02$ & $8.11 \mathrm{E}-02$ & $8.11 \mathrm{E}-02$ & $8.11 \mathrm{E}-02$ & $8.11 \mathrm{E}-02$ & $8.11 \mathrm{E}-02$ \\
\hline $8.00 \mathrm{E}-01$ & $6.67 \mathrm{E}-02$ & $6.66 \mathrm{E}-02$ & $6.66 \mathrm{E}-02$ & 6.67E-02 & $6.66 \mathrm{E}-02$ & $6.67 \mathrm{E}-02$ \\
\hline $1.00 \mathrm{E}+00$ & 5.81E-02 & $5.80 \mathrm{E}-02$ & $5.80 \mathrm{E}-02$ & $5.81 \mathrm{E}-02$ & $5.80 \mathrm{E}-02$ & $5.81 \mathrm{E}-02$ \\
\hline $1.02 \mathrm{E}+00$ & $5.74 \mathrm{E}-02$ & $5.73 \mathrm{E}-02$ & $5.73 \mathrm{E}-02$ & $5.74 \mathrm{E}-02$ & $5.73 \mathrm{E}-02$ & $5.74 \mathrm{E}-02$ \\
\hline $1.25 \mathrm{E}+00$ & $5.10 \mathrm{E}-02$ & $5.10 \mathrm{E}-02$ & $5.10 \mathrm{E}-02$ & $5.10 \mathrm{E}-02$ & $5.10 \mathrm{E}-02$ & $5.10 \mathrm{E}-02$ \\
\hline $1.50 \mathrm{E}+00$ & 4.65E-02 & 4.64E-02 & 4.64E-02 & 4.64E-02 & 4.64E-02 & 4.64E-02 \\
\hline $2.00 \mathrm{E}+00$ & $4.12 \mathrm{E}-02$ & $4.12 \mathrm{E}-02$ & $4.12 \mathrm{E}-02$ & $4.12 \mathrm{E}-02$ & $4.12 \mathrm{E}-02$ & 4.12E-02 \\
\hline $2.04 \mathrm{E}+00$ & 4.09E-02 & $4.09 \mathrm{E}-02$ & $4.09 \mathrm{E}-02$ & $4.09 \mathrm{E}-02$ & $4.09 \mathrm{E}-02$ & 4.09E-02 \\
\hline $3.00 \mathrm{E}+00$ & 3.69E-02 & $3.69 \mathrm{E}-02$ & $3.69 \mathrm{E}-02$ & $3.69 \mathrm{E}-02$ & $3.69 \mathrm{E}-02$ & 3.69E-02 \\
\hline $4.00 \mathrm{E}+00$ & $3.56 \mathrm{E}-02$ & $3.56 \mathrm{E}-02$ & $3.56 \mathrm{E}-02$ & $3.56 \mathrm{E}-02$ & $3.56 \mathrm{E}-02$ & $3.56 \mathrm{E}-02$ \\
\hline $5.00 \mathrm{E}+00$ & $3.54 \mathrm{E}-02$ & $3.55 \mathrm{E}-02$ & $3.55 \mathrm{E}-02$ & $3.55 \mathrm{E}-02$ & $3.55 \mathrm{E}-02$ & $3.55 \mathrm{E}-02$ \\
\hline $6.00 \mathrm{E}+00$ & $3.58 \mathrm{E}-02$ & $3.58 \mathrm{E}-02$ & $3.58 \mathrm{E}-02$ & $3.58 \mathrm{E}-02$ & $3.58 \mathrm{E}-02$ & $3.58 \mathrm{E}-02$ \\
\hline $7.00 \mathrm{E}+00$ & $3.64 \mathrm{E}-02$ & 3.64E-02 & 3.64E-02 & 3.64E-02 & $3.64 \mathrm{E}-02$ & 3.64E-02 \\
\hline $8.00 \mathrm{E}+00$ & $3.72 \mathrm{E}-02$ & $3.72 \mathrm{E}-02$ & 3.72E-02 & $3.72 \mathrm{E}-02$ & $3.72 \mathrm{E}-02$ & 3.72E-02 \\
\hline $9.00 \mathrm{E}+00$ & $3.80 \mathrm{E}-02$ & $3.80 \mathrm{E}-02$ & $3.80 \mathrm{E}-02$ & $3.80 \mathrm{E}-02$ & $3.80 \mathrm{E}-02$ & $3.80 \mathrm{E}-02$ \\
\hline $1.00 \mathrm{E}+01$ & $3.88 \mathrm{E}-02$ & $3.89 \mathrm{E}-02$ & $3.89 \mathrm{E}-02$ & $3.89 \mathrm{E}-02$ & $3.89 \mathrm{E}-02$ & $3.89 \mathrm{E}-02$ \\
\hline
\end{tabular}




\begin{tabular}{|c|c|c|c|c|c|c|}
\hline Photon Energy(MeV) & Sn 2.0Ag0.5Cu & Sn 2.0Ag1.5Cu & Sn 2.0Ag1.5Cu & Sn 3.5Ag0.8Cu & Sn 3.4Ag0.9Cu & Sn 3.0Ag0.9Cu \\
\hline $1.00 \mathrm{E}-02$ & $1.38 \mathrm{E}+02$ & $1.39 \mathrm{E}+02$ & $1.38 \mathrm{E}+02$ & $1.38 \mathrm{E}+02$ & $1.39 \mathrm{E}+02$ & $1.39 \mathrm{E}+02$ \\
\hline $1.50 \mathrm{E}-02$ & $4.67 \mathrm{E}+01$ & $4.69 \mathrm{E}+01$ & $4.66 \mathrm{E}+01$ & $4.66 \mathrm{E}+01$ & $4.67 \mathrm{E}+01$ & $4.67 \mathrm{E}+01$ \\
\hline $2.00 \mathrm{E}-02$ & $2.15 \mathrm{E}+01$ & $2.16 \mathrm{E}+01$ & $2.15 \mathrm{E}+01$ & $2.15 \mathrm{E}+01$ & $2.15 \mathrm{E}+01$ & $2.15 \mathrm{E}+01$ \\
\hline $2.55 \mathrm{E}-02$ & $1.11 \mathrm{E}+01$ & $1.12 \mathrm{E}+01$ & $1.11 \mathrm{E}+01$ & $1.11 \mathrm{E}+01$ & $1.11 \mathrm{E}+01$ & $1.11 \mathrm{E}+01$ \\
\hline 2.73E-02 & $1.01 \mathrm{E}+01$ & $1.01 \mathrm{E}+01$ & $1.06 \mathrm{E}+01$ & $1.06 \mathrm{E}+01$ & $1.06 \mathrm{E}+01$ & $1.05 \mathrm{E}+01$ \\
\hline 2.92E-02 & $8.41 \mathrm{E}+00$ & $8.45 \mathrm{E}+00$ & $8.90 \mathrm{E}+00$ & $8.90 \mathrm{E}+00$ & $8.87 \mathrm{E}+00$ & $8.74 \mathrm{E}+00$ \\
\hline $3.00 \mathrm{E}-02$ & $4.10 \mathrm{E}+01$ & $4.07 \mathrm{E}+01$ & $4.08 \mathrm{E}+01$ & $4.08 \mathrm{E}+01$ & $4.08 \mathrm{E}+01$ & $4.08 \mathrm{E}+01$ \\
\hline $4.00 \mathrm{E}-02$ & $1.93 \mathrm{E}+01$ & $1.92 \mathrm{E}+01$ & $1.92 \mathrm{E}+01$ & $1.92 \mathrm{E}+01$ & $1.92 \mathrm{E}+01$ & $1.92 \mathrm{E}+01$ \\
\hline $5.00 \mathrm{E}-02$ & $1.06 \mathrm{E}+01$ & $1.06 \mathrm{E}+01$ & $1.06 \mathrm{E}+01$ & $1.06 \mathrm{E}+01$ & $1.06 \mathrm{E}+01$ & $1.06 \mathrm{E}+01$ \\
\hline $6.00 \mathrm{E}-02$ & $6.52 \mathrm{E}+00$ & $6.48 \mathrm{E}+00$ & $6.50 \mathrm{E}+00$ & $6.50 \mathrm{E}+00$ & $6.49 \mathrm{E}+00$ & $6.50 \mathrm{E}+00$ \\
\hline $8.00 \mathrm{E}-02$ & $3.01 \mathrm{E}+00$ & $2.99 \mathrm{E}+00$ & $3.00 \mathrm{E}+00$ & $3.00 \mathrm{E}+00$ & $3.00 \mathrm{E}+00$ & $3.00 \mathrm{E}+00$ \\
\hline $1.00 \mathrm{E}-01$ & $1.67 \mathrm{E}+00$ & $1.65 \mathrm{E}+00$ & $1.66 \mathrm{E}+00$ & $1.66 \mathrm{E}+00$ & $1.66 \mathrm{E}+00$ & $1.66 \mathrm{E}+00$ \\
\hline $1.50 \mathrm{E}-01$ & $6.06 \mathrm{E}-01$ & $6.02 \mathrm{E}-01$ & $6.04 \mathrm{E}-01$ & $6.04 \mathrm{E}-01$ & $6.03 \mathrm{E}-01$ & $6.04 \mathrm{E}-01$ \\
\hline $2.00 \mathrm{E}-01$ & $3.25 \mathrm{E}-01$ & $3.23 \mathrm{E}-01$ & 3.24E-01 & $3.24 \mathrm{E}-01$ & $3.24 \mathrm{E}-01$ & $3.24 \mathrm{E}-01$ \\
\hline $3.00 \mathrm{E}-01$ & $1.64 \mathrm{E}-01$ & $1.63 \mathrm{E}-01$ & $1.63 \mathrm{E}-01$ & $1.63 \mathrm{E}-01$ & $1.63 \mathrm{E}-01$ & 1.63E-01 \\
\hline $4.00 \mathrm{E}-01$ & $1.15 \mathrm{E}-01$ & $1.15 \mathrm{E}-01$ & $1.15 \mathrm{E}-01$ & $1.15 \mathrm{E}-01$ & $1.15 \mathrm{E}-01$ & $1.15 \mathrm{E}-01$ \\
\hline $5.00 \mathrm{E}-01$ & $9.37 \mathrm{E}-02$ & $9.36 \mathrm{E}-02$ & $9.36 \mathrm{E}-02$ & $9.36 \mathrm{E}-02$ & $9.36 \mathrm{E}-02$ & $9.36 \mathrm{E}-02$ \\
\hline $6.00 \mathrm{E}-01$ & $8.11 \mathrm{E}-02$ & $8.11 \mathrm{E}-02$ & $8.11 \mathrm{E}-02$ & $8.11 \mathrm{E}-02$ & $8.11 \mathrm{E}-02$ & $8.11 \mathrm{E}-02$ \\
\hline $8.00 \mathrm{E}-01$ & $6.66 \mathrm{E}-02$ & $6.66 \mathrm{E}-02$ & $6.67 \mathrm{E}-02$ & 6.67E-02 & $6.67 \mathrm{E}-02$ & 6.67E-02 \\
\hline $1.00 \mathrm{E}+00$ & $5.80 \mathrm{E}-02$ & $5.80 \mathrm{E}-02$ & $5.81 \mathrm{E}-02$ & $5.81 \mathrm{E}-02$ & $5.81 \mathrm{E}-02$ & $5.81 \mathrm{E}-02$ \\
\hline $1.02 \mathrm{E}+00$ & $5.73 \mathrm{E}-02$ & $5.73 \mathrm{E}-02$ & $5.74 \mathrm{E}-02$ & $5.74 \mathrm{E}-02$ & $5.74 \mathrm{E}-02$ & $5.73 \mathrm{E}-02$ \\
\hline $1.25 \mathrm{E}+00$ & $5.10 \mathrm{E}-02$ & $5.10 \mathrm{E}-02$ & $5.10 \mathrm{E}-02$ & $5.10 \mathrm{E}-02$ & $5.10 \mathrm{E}-02$ & $5.10 \mathrm{E}-02$ \\
\hline $1.50 \mathrm{E}+00$ & 4.64E-02 & 4.64E-02 & 4.64E-02 & $4.64 \mathrm{E}-02$ & 4.64E-02 & $4.64 \mathrm{E}-02$ \\
\hline $2.00 \mathrm{E}+00$ & $4.11 \mathrm{E}-02$ & 4.12E-02 & 4.12E-02 & 4.12E-02 & $4.12 \mathrm{E}-02$ & 4.12E-02 \\
\hline $2.04 \mathrm{E}+00$ & 4.09E-02 & 4.09E-02 & 4.09E-02 & 4.09E-02 & 4.09E-02 & 4.09E-02 \\
\hline $3.00 \mathrm{E}+00$ & 3.69E-02 & $3.69 \mathrm{E}-02$ & 3.69E-02 & $3.69 \mathrm{E}-02$ & $3.69 \mathrm{E}-02$ & 3.69E-02 \\
\hline $4.00 \mathrm{E}+00$ & $3.56 \mathrm{E}-02$ & $3.56 \mathrm{E}-02$ & $3.56 \mathrm{E}-02$ & $3.56 \mathrm{E}-02$ & $3.56 \mathrm{E}-02$ & $3.56 \mathrm{E}-02$ \\
\hline $5.00 \mathrm{E}+00$ & $3.55 \mathrm{E}-02$ & $3.54 \mathrm{E}-02$ & $3.55 \mathrm{E}-02$ & $3.55 \mathrm{E}-02$ & $3.55 \mathrm{E}-02$ & $3.55 \mathrm{E}-02$ \\
\hline $6.00 \mathrm{E}+00$ & $3.58 \mathrm{E}-02$ & $3.58 \mathrm{E}-02$ & $3.58 \mathrm{E}-02$ & $3.58 \mathrm{E}-02$ & $3.58 \mathrm{E}-02$ & $3.58 \mathrm{E}-02$ \\
\hline $7.00 \mathrm{E}+00$ & 3.64E-02 & 3.64E-02 & 3.64E-02 & 3.64E-02 & 3.64E-02 & 3.64E-02 \\
\hline $8.00 \mathrm{E}+00$ & $3.72 \mathrm{E}-02$ & $3.71 \mathrm{E}-02$ & 3.72E-02 & $3.72 \mathrm{E}-02$ & $3.72 \mathrm{E}-02$ & 3.72E-02 \\
\hline $9.00 \mathrm{E}+00$ & $3.80 \mathrm{E}-02$ & $3.80 \mathrm{E}-02$ & $3.80 \mathrm{E}-02$ & $3.80 \mathrm{E}-02$ & $3.80 \mathrm{E}-02$ & $3.80 \mathrm{E}-02$ \\
\hline $1.00 \mathrm{E}+01$ & $3.89 \mathrm{E}-02$ & $3.88 \mathrm{E}-02$ & $3.89 \mathrm{E}-02$ & $3.89 \mathrm{E}-02$ & $3.89 \mathrm{E}-02$ & $3.89 \mathrm{E}-02$ \\
\hline Photon Energy(MeV) & Sn $2.0 \mathrm{Ag} 0.9 \mathrm{Cu}$ & Sn 4.0Ag1.5Cu & Sn 3.5Ag0.5Cu & Sn 3.5Ag2.0Cu & $\mathbf{P b}$ & \\
\hline $1.00 \mathrm{E}-02$ & $1.39 \mathrm{E}+02$ & $1.39 \mathrm{E}+02$ & $1.39 \mathrm{E}+02$ & $1.39 \mathrm{E}+02$ & $1.31 \mathrm{E}+02$ & \\
\hline $1.50 \mathrm{E}-02$ & $4.68 \mathrm{E}+01$ & $4.68 \mathrm{E}+01$ & 4.67E+01 & $4.70 \mathrm{E}+01$ & $1.12 \mathrm{E}+02$ & \\
\hline $2.00 \mathrm{E}-02$ & $2.15 \mathrm{E}+01$ & $2.15 \mathrm{E}+01$ & $2.15 \mathrm{E}+01$ & $2.16 \mathrm{E}+01$ & $\underline{8.64 \mathrm{E}+01}$ & \\
\hline $2.55 \mathrm{E}-02$ & $1.12 \mathrm{E}+01$ & $1.12 \mathrm{E}+01$ & $1.11 \mathrm{E}+01$ & $1.12 \mathrm{E}+01$ & $4.61 \mathrm{E}+01$ & \\
\hline $2.73 \mathrm{E}-02$ & $1.01 \mathrm{E}+01$ & $1.09 \mathrm{E}+01$ & $1.06 \mathrm{E}+01$ & $1.07 \mathrm{E}+01$ & $3.86 \mathrm{E}+01$ & \\
\hline
\end{tabular}




\begin{tabular}{|c|c|c|c|c|c|}
\hline $2.92 \mathrm{E}-02$ & $8.43 \mathrm{E}+00$ & $9.08 \mathrm{E}+00$ & $8.90 \mathrm{E}+00$ & $8.94 \mathrm{E}+00$ & $3.25 \mathrm{E}+01$ \\
\hline $3.00 \mathrm{E}-02$ & $4.09 \mathrm{E}+01$ & $4.06 \mathrm{E}+01$ & $4.08 \mathrm{E}+01$ & $4.05 \mathrm{E}+01$ & $3.03 \mathrm{E}+01$ \\
\hline $4.00 \mathrm{E}-02$ & $1.93 \mathrm{E}+01$ & $1.91 \mathrm{E}+01$ & $1.92 \mathrm{E}+01$ & $1.91 \mathrm{E}+01$ & $1.44 \mathrm{E}+01$ \\
\hline $5.00 \mathrm{E}-02$ & $1.06 \mathrm{E}+01$ & $1.05 \mathrm{E}+01$ & $1.06 \mathrm{E}+01$ & $1.05 \mathrm{E}+01$ & $8.04 \mathrm{E}+00$ \\
\hline $6.00 \mathrm{E}-02$ & $6.50 \mathrm{E}+00$ & $6.46 \mathrm{E}+00$ & $6.49 \mathrm{E}+00$ & $6.44 \mathrm{E}+00$ & $5.02 \mathrm{E}+00$ \\
\hline $8.00 \mathrm{E}-02$ & $3.00 \mathrm{E}+00$ & $2.98 \mathrm{E}+00$ & $2.99 \mathrm{E}+00$ & $2.97 \mathrm{E}+00$ & $2.42 \mathrm{E}+00$ \\
\hline $1.00 \mathrm{E}-01$ & $1.66 \mathrm{E}+00$ & $1.65 \mathrm{E}+00$ & $1.66 \mathrm{E}+00$ & $1.65 \mathrm{E}+00$ & $5.55 \mathrm{E}+00$ \\
\hline $1.50 \mathrm{E}-01$ & $6.04 \mathrm{E}-01$ & $6.01 \mathrm{E}-01$ & $6.03 \mathrm{E}-01$ & $5.99 \mathrm{E}-01$ & $2.02 \mathrm{E}+00$ \\
\hline $2.00 \mathrm{E}-01$ & 3.24E-01 & $3.22 \mathrm{E}-01$ & $3.23 \mathrm{E}-01$ & $3.22 \mathrm{E}-01$ & $9.99 \mathrm{E}-01$ \\
\hline $3.00 \mathrm{E}-01$ & $1.63 \mathrm{E}-01$ & $1.63 \mathrm{E}-01$ & 1.63E-01 & $1.63 \mathrm{E}-01$ & 4.03E-01 \\
\hline $4.00 \mathrm{E}-01$ & $1.15 \mathrm{E}-01$ & $1.15 \mathrm{E}-01$ & $1.15 \mathrm{E}-01$ & $1.15 \mathrm{E}-01$ & $2.32 \mathrm{E}-01$ \\
\hline $5.00 \mathrm{E}-01$ & $9.36 \mathrm{E}-02$ & $9.36 \mathrm{E}-02$ & $9.36 \mathrm{E}-02$ & $9.35 \mathrm{E}-02$ & $1.61 \mathrm{E}-01$ \\
\hline $6.00 \mathrm{E}-01$ & 8.11E-02 & $8.11 \mathrm{E}-02$ & $8.11 \mathrm{E}-02$ & $8.11 \mathrm{E}-02$ & $1.25 \mathrm{E}-01$ \\
\hline $8.00 \mathrm{E}-01$ & $6.66 \mathrm{E}-02$ & 6.67E-02 & 6.67E-02 & 6.67E-02 & 8.87E-02 \\
\hline $1.00 \mathrm{E}+00$ & $5.80 \mathrm{E}-02$ & $5.81 \mathrm{E}-02$ & $5.81 \mathrm{E}-02$ & $5.81 \mathrm{E}-02$ & $7.10 \mathrm{E}-02$ \\
\hline $1.02 \mathrm{E}+00$ & $5.73 \mathrm{E}-02$ & $5.74 \mathrm{E}-02$ & $5.74 \mathrm{E}-02$ & $5.74 \mathrm{E}-02$ & $6.98 \mathrm{E}-02$ \\
\hline $1.25 \mathrm{E}+00$ & $5.10 \mathrm{E}-02$ & $5.10 \mathrm{E}-02$ & $5.10 \mathrm{E}-02$ & $5.10 \mathrm{E}-02$ & $5.88 \mathrm{E}-02$ \\
\hline $1.50 \mathrm{E}+00$ & 4.64E-02 & 4.65E-02 & 4.64E-02 & 4.65E-02 & $5.22 \mathrm{E}-02$ \\
\hline $2.00 \mathrm{E}+00$ & 4.12E-02 & $4.12 \mathrm{E}-02$ & $4.12 \mathrm{E}-02$ & $4.12 \mathrm{E}-02$ & 4.61E-02 \\
\hline $2.04 \mathrm{E}+00$ & $4.09 \mathrm{E}-02$ & 4.09E-02 & 4.09E-02 & 4.09E-02 & $4.58 \mathrm{E}-02$ \\
\hline $3.00 \mathrm{E}+00$ & $3.69 \mathrm{E}-02$ & $3.69 \mathrm{E}-02$ & $3.69 \mathrm{E}-02$ & $3.69 \mathrm{E}-02$ & 4.23E-02 \\
\hline $4.00 \mathrm{E}+00$ & $3.56 \mathrm{E}-02$ & $3.56 \mathrm{E}-02$ & $3.56 \mathrm{E}-02$ & $3.56 \mathrm{E}-02$ & $4.20 \mathrm{E}-02$ \\
\hline $5.00 \mathrm{E}+00$ & $3.55 \mathrm{E}-02$ & $3.54 \mathrm{E}-02$ & $3.55 \mathrm{E}-02$ & $3.54 \mathrm{E}-02$ & $4.27 \mathrm{E}-02$ \\
\hline $6.00 \mathrm{E}+00$ & $3.58 \mathrm{E}-02$ & $3.58 \mathrm{E}-02$ & $3.58 \mathrm{E}-02$ & $3.57 \mathrm{E}-02$ & $4.39 \mathrm{E}-02$ \\
\hline $7.00 \mathrm{E}+00$ & $3.64 \mathrm{E}-02$ & $3.64 \mathrm{E}-02$ & 3.64E-02 & 3.64E-02 & $4.53 \mathrm{E}-02$ \\
\hline $8.00 \mathrm{E}+00$ & $3.72 \mathrm{E}-02$ & $3.71 \mathrm{E}-02$ & $3.72 \mathrm{E}-02$ & $3.71 \mathrm{E}-02$ & $4.68 \mathrm{E}-02$ \\
\hline $9.00 \mathrm{E}+00$ & $3.80 \mathrm{E}-02$ & $3.80 \mathrm{E}-02$ & $3.80 \mathrm{E}-02$ & $3.79 \mathrm{E}-02$ & 4.82E-02 \\
\hline $1.00 \mathrm{E}+01$ & $3.89 \mathrm{E}-02$ & $3.88 \mathrm{E}-02$ & $3.89 \mathrm{E}-02$ & $3.88 \mathrm{E}-02$ & 4.97E-02 \\
\hline
\end{tabular}

Figure 2 is illustrated the data of the mass absorption coefficients of the highest alloy density (Ag3.8 Cu0.7 Sn95.5) and (Ag3.5 Cu0.75 Sn95.75), lowest alloy density (Ag2.0 Cu0.5 Sn97.5) and (Ag2.0Cu0.9Sn97.1) and lead (Pb). We can conclude from Figure 2 that the investigated alloys provide approximately similar attenuation of the traditional shielding materials (lead) and as the energies of gamma- ray increase the mass attenuation coefficients $\left(\mu_{\rho}\right)$ decrease for all investigated materials. 


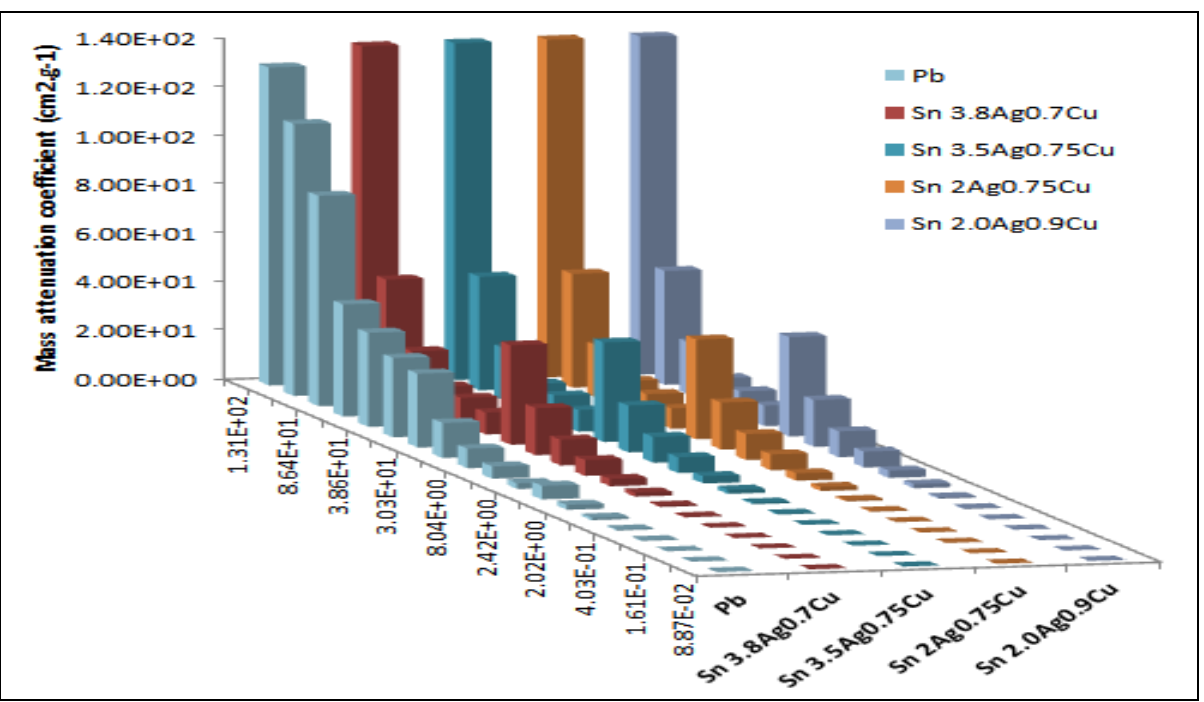

Figure 2. Mass absorption coefficient versus gamma radiation energy for investigated alloy

\section{Conclusions}

In this work a new material has been tested for radiation shielding applications, in order to meet needs for lightweight multifunctional shielding materials. The results reveal that the investigated alloys $\mathrm{Ag}-\mathrm{Cu}-\mathrm{Sn}$ provided similar protection as pure Lead $(\mathrm{Pb})$ per unit mass, in most cases. Findings obtained in this study showed that, if (Ag-Cu-Sn) alloys used as a radiation shielding, its advantages are:

1. (Ag-Cu-Sn) alloys present a slightly increase in absorbing of low photon energy compared to lead $(\mathrm{Pb})$.

2. It is lighter than traditional material shield Lead $(\mathrm{Pb})$.

3. Its melting temperatures are between $\left(203\right.$ to $\left.221^{\circ} \mathrm{C}\right)$ lower than Lead $\left(327^{\circ} \mathrm{C}\right)$.

\section{References}

[1] N. Tsoulfanidis, "Measurement and Detection of radiation", $2^{\text {nd }}$ edition, (1995).

[2] S. Xu, "A Novel Ultra-light Structure for Radiation Shielding", MsD thesis, Graduate Faculty of North Carolina State University, (2008).

[3] J. K. Shultis and R. E. Faw, "Radiation Shielding", Dept. of Nuclear Engineering, Kansas State Univ., Manhattan, Kansas, (2000).

[4] W. D. Ehmann and D. E. Vance, "Radiochemistry and Nuclear Methods of Analysis", John Wiley and Sons, New York, (1991), pp. 162-175.

[5] G. Nelson and D. Reilly "Gamma-Ray Interactions with Matter", in Passive Nondestructive Analysis of Nuclear Materials, Los Alamos National Laboratory, NUREG/CR-5550, LA-UR-90-732, (1991), pp. $27-42$.

[6] G. F. Knoll, "Radiation Detection and Measurement", $3^{\text {rd }}$ edition, (2000).

[7] E. W. Webster, "Experiments with medium Z materials for shielding against low-energy x-rays", Radiology, vol. 86, (1966), pp. 146.

[8] J. P. McCaffrey, H. Shen, B. Downton and E. Mainegra-Hing, "Radiation attenuation by lead and nonlead materials used in radiation shielding garments", Med. Phys., vol. 34, no. 2, (2007) February.

[9] National Istitute of Standard and Technology, Physical Measurements Laboratory, XCOM Photon CrossSections Database, http://physics.nist.gov/PhysRefData/Xcom/ html/xcom1.html.

[10] A. Mikula and H. Ipser, "Database for Properties of Lead-Free Solder Alloys", Version 1.0, (2006).

[11] handymath.com, "Solutions For Technicians", http://www.handymath.com/cgi-bin/density.cgi. 
International Journal of Advanced Science and Technology Vol.63, (2014) 